returned and fell ill with influenza; strict isolation was even then successful in preventing its spread to the inmates.

Dr. Caldwell Smith's evidence as to ætiology is valuable and interesting. "It is to the life history of Pfeiffer's bacillus that we must direct our attention if we wish to understand the seemingly strange vagaries of the disease. An individual is infected by breathing at once the expired air from a person suffering from the disease, and I believe this to be the only method of infection."

The concourse of people is favourable to the spread of influenza in two ways, according to Dr. Parsons : firstly, hy bringing the affected and the healthy near together; and secondly, by the poison being present in a more concentrated form in confined and vitiated air.

Among the discussions which throw light on the character of the disease, and bear upon the means of prevention, the following may be mentioned: on the degree of protection afforded to individuals and to communities by previous attacks, on the influence of occupation and of unsanitary conditions, on the connection with pneumonia, on the period of infectiousness, on the clinical features of the later epidemics, and on relapses.

The researches of Dr. Klein, in respect to the effect of inocula. tion upon animals, gave results for the most part negative. His affirmative results, however, were "in full agreement with the results obtained by Pfeiffer and Kitasato." The bacillus was always abundantly present in the bronchial secretion of patients suffering from influenza, diminishing in number as the disease abated.

"It is to be feared," wrote Dr. Parsons, "that the contagion of influenza is still domiciled among us, and that a renewal of its epidemic activity within the next few years is by no mean improbable." The expected revival is now only too apparent. A certain proverb declares, with the rashness of its class, that the man once bit is twice shy. In a literal sense, the saying may contain a good deal of truth, but to nations, or aggregations of individuals, it is quite inapplicable. The development of common sense for common action against these evils has still to take place. This country has now passed through three severe epidemics of influenza within four years, each outbreak drawing many sad maladies in its train, prostrating hundreds of thousands of breadwinners, cutting short many illustrious lives, and crippling many for years to come, and we are now running into a fourth epidemic in London, without any great organised attempt being made to counteract it.

The provisional memorandum of the Local Government Board, issued on January 23,1892 , impressed upon the public the fact that in its epidemic form influenza is an eminently infectious complaint, communicable in the ordinary personal relations of individuals with each other, that separation of the sick from the healthy should as far as practicable be carried out, that rooms, \&c. should be disinfected, and that ventilation should receive special attention.

It would be some defence against a serious recrudescence of the pest if this memorandum, or an abstract of it, were supplied to every householder on the first threatenings of an outbreak in any locality. In his article on prophylaxis, $\mathrm{Dr}$. Parsons remarks on the difficulties which would frustrate any measures of notification and isolation on a large scale, but suggests that notification, with fees for early cases only, might be tried in certain districts, and that such a measure should be adopted "in the interval before another epidemic." So much experience has been gained in distinguishing the symptoms of influenza from those of other ailments, that the difficulty of diagnosis cannot now be an insuperable bar to attempts at prevention. It is well to remember that the pecuniary cost of prevention cannot be compared with the loss to the country by an epidemic, for this has been proved to amount to millions.

Among places and means of infection which may cause much mischief, but are not noticed in this volume, are bakers' shops, in which the baker or attendant suffers from influenza or severe cold; booking offices, post offices, banks, \&c. in which the mouth and the ledger, \&c. are in multiple communication; letters written and fastened by patients; and, most of all, railway carriages packed full and with windows closed, daily conveying vast numbers of people to and from the city, and containing perhaps the most organically polluted air which can easily be found in a civilised country.

The report closes with an interesting statement respecting the immunity of animals, including monkeys, at the Zoologica Gardens.

R. RUSSELL.

\section{ON A METHOD OF SEPARATING THE} MINERAL COMPONENTS OF A ROCK.

$\mathrm{T}$ is told of a famous German petrographer, that whenever appealed to by a student in difficulties over a problematical mineral in a rock-slice, his invariable advice was "Get it out."

It is hard dispassionately to reflect on the sufferings to which this simple process of "getting it out" have given rise. All we petrographers have passed through the vale! May we now indulge the pious hope that the following simple apparatus may bring some mitigation to the ordeal? It will certainly save a good deal of time and trouble when only small quantities. of a particular mineral are required ; enough, that is, for a blow. pipe analysis, a flame test, and microscopical examination.

A large test-tube (see Fig.), conveniently six inches in length by three-quarters in diameter, is filled with heavy solution, graded from specific gravity 3.3 to 2.5 , so as to form after standing a diffusion column, as already described in NATURE, vol. xliii. p. 404, 189r. It is not necessary to wait till the change in density of the column is uniform from top to bottom; by introducing a sufficient number of specific gravity indexes the column is mapped out into a succession of lengths, within the limits of each of which the change of density is practically uniform, certainly sufficiently so for mineral determinations.

A fragment of the rock to be examined, about the size of a hazel-nut, is powdered in the usual way, sifted and washed, dried and then introduced into the diffusion column. Separation of the constituent minerals at once begins to take place, and in the course of a few hours is complete. Each species of mineral is then floating in liquid of its own specific gravity; the next problem is to get it out. A pipette as commonly used is not sufficient, for as it is introduced grains of minerals from other zones than that sought for, adhere to its sides; on removing the finger, the sudden inrush of fluid carries with it grains from surrounding zones, and finally on drawing up the pipette, fluids of zones lying above that to which it has descended displace the heavier fluid it already contains, carrying with them suspended grains, and thus bringing about the mixture which it is our desire to prevent.

With very little trouble these difficulties may be completely overcome. To prevent the sudden inrush of fluid the pipette, which should be of small calibre (in my experiments it measures $1.5 \mathrm{~mm}$.), is fitted with a piston $(p)$. This may be very simply made by winding a little unravelled cotton thread round the end of a stem of Esparto grass, such as is sold for cleaning tobaccopipes. The piston is pushed down to the bottom of the pipette, which is then ready for use.

To extract grains from any zone the pipette is slid down into the diffusion column till its lower end is just immersed in the zone; a gentle shake given to it as it passes through the solution will serve to detach adhering particles; the piston is then slowly raised, and the fluid with its floating mineral grains quietly follows it, the other zones remaining undisturbed. To prevent the fluid of higher zones entering the pipette as it is withdrawn, it is necessary to plug its lower end ; no very tight closure is necessary, since the piston, which now lies at the upper end of the pipette, by excluding the air ensures the retention of the contained column of fluid; all that is needed is a stopper, which will exclude solid particles. A very thin glass rod is rounded off at one end, which is then bent upwards into the form of a crook $(c)$. The crook is let down into the diffusion column till its upward pointing lower end lies beneath the open extremity of the pipette, which it completely blocks up on being 
raised into position. The pipette with the crook is then taken out of the fluid, and inverted. The crook is laid aside, and the outside of the pipette cleaned with blotting-paper, by which all adhering foreign grains are removed. The pipette now con tains a pure gathering of the mineral required, and it only remains to discharge its contents, and this is of course accomplished by pushing down the piston.

Minerals may thus be removed from every zone of a diffusion column; and all the species which enter into the composition of a rock, except of course the very heaviest, may be separately obtained, with their specific gravity determined as an incident of the process.

In this absurdly simple fashion "getting it out" ceases to be a penance, and becomes a pleasure. W. J. Sollas.

\section{THE CLOUDY CONDENSATION OF STEAM. ${ }^{1}$}

THE air, as every one knows, is composed almost entirely of the two gases, oxygen and nitrogen. It also contains small quantities of other substances, of which the chief are carsonic acid gas and water vapour, and it is the latter of these constituents, water vapour, or "steam," as it is sometimes called, that will principally concern us this evening.

The quantity of invisible water vapour which the air can at any time take up depends upon the temperature ; the higher the temperature of the air the more water it can contain. The proportion. however, never exceeds a few grains' weight of water to a cubic foot of air. Air at any temperature, containing as much water as it can pcsibly hold, is said to be "saturated," while the temperature at which air containing a certain proportion of water becomes saturated is called the "dew point."

The large glass globe, upon which the beam from the electric lantern is now directed, contains ordinary air, kept in a state of saturation, or nearly so, by the presence of a little water. You will observe that although heavily laden with water vapour the air is perfectly transparent. If, now, we turn a tap, and so connect the globe with an exhausted receiver, the air expands and becomes colder; the space inside the globe is no longer able to hold tne same quantity of water as before in the form of vapour, and the excess is precipitated as very finely divided liquid water, which fills the globe and is perfectly visible as a cloud or mist. In a few minutes the cloud disappears, partly, no doubt, because some of the particles of water have fallen to the bottom of the vessel, but chiefly because the air becomes in time warmed up to its original temperature (that of the room), and the suspended water is converted back again into invisible vapour.

I once more rarefy the air, and admit a fresh supply while holding the flame of a spirit lamp near the orifice of the inlet pipe, so that some of the burnt air is carried into the interior of the globe. When the air is again expanded a cloud is formed which is far more dense than the others were. It appears on examination that the increased density of this cloud is not due to the condensation of a greater quantity of water. Little, if any, more water is precipitated than before. But the water particles are now much more numerous, their increased number being compensated for by diminished size. Within certain limits, the greater the number of particles into which a given quantity of water is condensed, the greater will be the apparent thickness of the mist produced. A few large drops will not impede and scatter light to the same extent as a great number of small ones, though the actual quantity of condensed water may be the same in each case.

Then comes the question, why should the burnt air from the flame so greatly increase the number of the condensed drops ? An answer, though perhaps not quite a complete one, is furnished by some remarkable experiments made by M. Coulier, a French pro fessor, nearly twenty years ago. He believed his experiments showed that water vapour would not condense at all, even at temperatures far below the dew point, unless there were present in the air a number of material particles to serve as nuclei around which the condensation would take place. All air, he says, contains dust; and anything that increases the number of dust particles in the air increases the density of the condensation by affording a greater number of nuclei. Air in which a flame had been burnt he supposed to be very highly charged with finely divided matter, the products of combustion,

I Extracted from a lecture on " Fogs, Clouds, and Lightning," delivered at the Royal Institution on May 5 .

NO. I 26 I, vOL. 49] and thus rendered extraordinarily " active" in bringing about condensation. And that, according to Coulier's view, is the reason why such a dense fog was formed when air which had been contaminated by the spirit flame was admitted to our globe.

On the other hand, air, even burnt air, which has been filtered through tightly packed cotton wool, is found to be perfectly inactive. No cloud or mist will form in it, however highly it may be supersaturated. Coulier explained this fact by supposing that the process of filtration completely removed all dust particles from the air.

The experiments of Coulier were repeated and confirmed by Mascart. The latter also made one additional observation which may very probably turn out to be of great importance. He found that ozone, or rather, strongly ozonised air, was a very active mist producer, and that unlike ordinary air, it was not deprived of its activity by filtration.

Four or five years later, all the facts which had been noticed by Coulier, and others of an allied nature, were independently discovered by Mr. Aitken, who has devoted much time and study to them, and made them a foundation of an entirely new branch of meteorology.

Later, perhaps, we may see reason to doubt whether all the conclusions of Coulier and Aitken are quite accurate, especially as regards the action of so-called products of combustion.

Every one has noticed how dense and dark a thundercloud is. It shuts out daylight almost as if it were a solid substance, and the glimmer that penetrates it is often imbued with a lurid or copper-coloured tint.

I had always found it rather difficult to believe that these peculiarities were due simply to the unusual extent and thickness of the clonds, as is commonly supposed to be the case, and it occurred to me about three years ago, that perhaps some clue to the explanation might be afforded by the electrification of a jet of steam. On making the experiment I found that the density and opacity of the jet were greatly increased when an electrical discharge was directed upon it, while its shadow, if cast upon a white screen by a sufficiently strong light, was of a decidedly reddish-brown tint.

As a possible explanation of the effect I suggested that there might occur some action among the little particles of water of a similar nature to that observed by Lord Rayleigh in his experiments upon water jets. A jet of water two or three feet long is made to issue in a nearly vertical direction from a small nozzle. At a certain distance above the nozzle the continuous stream is found to break up into separate drops, which coilide with one another, and again rebounding, become scattered over a considerable space. But when the jet is exposed to the influence of an electrified substance, such as a rubbed stick of sealing-wax, the drops no longer rebound after collision, but coalesce, and the entire stream of water, both ascending and descending, becomes nearly continuous.

There is one other point to which I wish to direct your particular attention. If the sealing-wax, or better, the knob of a charged Leyden jar, is held very close to the jet, so that the electrical influence is stronger, the separate drops do not coalesce as before, but become scattered even more widely than when no electrical influence was operating. They become similarly electrified and, in accordance with the well-known law, repel one another.

We will now remove the water jet, and in its place put a little apparatus for producing a jet of steam. It consists of a halfpint tin bottle, through the cork of which passes a glass tube terminating in a nozzle. When the water in the bottle is made to boil, a jet of steam issues from the nozzle, and if we observe the shadow of the steam jet upon the screen we shall see that it is of feeble intensity and of a neutral tint, unaccompanied by any trace of decided colour. A bundle of needles connected by a wire with the electrical machine is placed near the base of the jet, and when the machine is worked electricity is discharged into the steam. A very striking effect instantly follows. The cloud of condensed steam is rendered dense and dark, its shadow at the same time assuming the suggestive yellowishbrown colour.

I at first believed that we had here a repetition, upon a smaller scale, of the phenomenon which occurs in the water jet. The little particles of condensed water must frequently come into collision with one another, and it seemed natural to suppose that, like Lord Rayleigh's larger particles, they rebounded under ordinary circumstances, and coalesced when under the influence 